\title{
Las ciudades medias históricas
}

Juan Luis Díaz Quidiello

Servicio de Planificación Regional

Consejería de Obras Públicas y Transportes. Junta de Andalucia

\section{Resumen:}

Las ciudades medias del interior de Andalucía son una singularidad del sistema urbano andaluz, un tipo de fenómeno urbano peculiarmente andaluz. Consideradas tradicionalmente agrociudades sin verdadero rango urbano se muestran hoy, sin embargo, como un patrimonio territorial y urbano de primer orden, tanto para mejorar los equilibrios internos de la región, como para mejorar la competitividad de la región.

Frente a las tendencias que están provocando la concentración del desarrollo económico y social en la franja del litoral y en las grandes aglomeraciones urbanas, este tipo de ciudades están llamadas a ser un contrapeso territorial, que permita extender a la totalidad de la región los procesos de desarrollo.

\section{Palabras clave:}

Écija / Planificación territorial / Ordenación del territorio / Ciudades medias / Agrociudades

\section{LAS CIUDADES MEDIAS ANDALUZAS}

Aunque la denominación "ciudad media" es una palabra ampliamente utilizada, en especial en la planificación territorial, pero también en otras materias, es igualmente cierto que en muchas ocasiones se trata de un concepto equívoco y casi siempre relativo. De hecho, el término alude en primer lugar a dimensiones, a tamaños, ya sean éstos poblacionales, económicos o funcionales y como tal su significado cambia según la escala y los ámbitos de que se trate. Así, cuando se toma como referencia ámbitos continentales, caso de la Unión Europea, es frecuente encontrar la denominación "ciudad media" referida a capitales de regiones alejadas de las grandes conurbaciones metropolitanas. Ciudades como SeviIla o Málaga, o más exactamente ellas más sus respectivas aglomeraciones urbanas, reciben a menudo esa denominación, cuando no otras de inferior nivel jerárquico.

Si se desciende, en cambio, a ámbitos más reducidos, caso de un Estado como España, el calificativo de media suele aplicarse a ciudades, muchas de ellas capitales de provincia, con algunos cientos de miles de habitantes y situadas fuera de los ámbitos de influencia directos de los grandes centros nacionales - Madrid y Barcelona, en España- o de las cabeceras regionales de mayor importancia. Capitales andaluzas como Granada, Cádiz, Huelva, Almería, Jaén, Córdoba, Jerez de la Frontera o Algeciras entran habitualmente dentro de este tipo cuando se trata de elaborar clasificaciones de ciudades que abarquen a la totalidad del territorio nacional.

En Andalucía, sin embargo, el término se ha reservado, y así lo recogen los documentos regionales de ordenación del territorio, al escalón inmediatamente inferior al formado por lo que se han dado en denominar las Ciudades Principales de la región, esto es, las ocho capitales de provincia más Jerez de la Frontera y Algeciras, que constituyen el primer nivel en la jerarquía urbana. Se identifican, de esta forma, las ciudades medias andaluzas con las poblaciones que ocupan una posición intermedia dentro de la red urbana regional entre las diez ciudades de mayor tamaño y rango funcional y los núcleos de población propiamente rurales.

Aunque es un error identificar exclusivamente los niveles de jerarquía de una ciudad con su tamaño de población, grosso modo esta primera aproximación implicaría identificar las ciudades medias de la región con aquellos núcleos urbanos situados aproximadamente entre los 20.000 y los 100.000 habitantes. Esto no significa, en cualquier caso, que no se pueda hablar de ciudades medias-siempre dentro de la escala regional andaluza- que no llegan a superar este umbral de población o, que en sentido contrario, algunas ciudades que sobrepasan los 100.000 habitantes no dejen de ser, por ello, ciudades medias.

Pero, como ya se ha apuntado, el volumen de población es sólo uno de los factores a tener en cuenta a la hora de identificar un asentamiento de población como una ciudad media. En realidad, son otros los rasgos que dan a una ciudad este carácter. Lo verdaderamente definitorio es el grado de centralidad que ha logrado alcanzar el núcleo en cuestión y el papel que desempeña en la estructura territorial de la región. La concentración de funciones supramunicipales de carácter intermedio, el protagonismo que tenga en los sistemas relacionales regionales o la forma en que participa en el tejido productivo de la región son, en última instancia, los elementos que hacen de una ciudad una ciudad media desde un punto de vista territorial y no necesariamente el número de residentes en ella. No son raros, en este sentido, los ejemplos de ciudades con una determinada población, que en la práctica, tienen un nivel funcional inferior al de otras ciudades de menor tamaño; y viceversa. 
Ahora bien, como ocurre habitualmente en todo sistema urbano regional, y con mayor claridad, aún, en el andaluz, el carácter medio que se asigna a un núcleo puede obedecer a una gran diversidad de situaciones y englobar hechos urbanos de muy diversa naturaleza. Una ciudad puede alcanzar la categoría de media gracias a una determinada función, ya sea ésta un servicio público o privado, una especial actividad económica o una posición singular en los sistemas de transportes o comunicaciones o, por el contrario, ser media por la simple acumulación de funciones no especializadas que ha alcanzado. Puede, igualmente, deber esta consideración a su pertenencia a una estructura supramunicipal -una aglomeración urbana, por ejemplo- de la que forma parte y en la que desempeña ese papel, o bien serlo por la dependencia que de ella tenga un ámbito rural extenso. Cabe, en definitiva, realizar múltiples clasificaciones de ciudades medias según el criterio que, en cada caso, se quiera tener en cuenta. Sí es importante tener siempre presente la complejidad de relaciones que se establecen dentro de un sistema urbano y que, casi nunca dentro de él las jerarquías se mantienen de forma rígida: una ciudad puede tener un nivel funcional determinado para un aspecto y otro, inferior o superior, para otro.

\section{CIUDADES MEDIAS METROPOLITANAS, CIUDADES MEDIAS LITORALES Y CIUDADES MEDIAS INTERIORES}

Una primera diferenciación por grandes ámbitos territoriales puede ayudar a acotar la diversidad de situaciones que se dan dentro de lo que se han dado en llamar ciudades medias andaluzas. Muy esquemáticamente pueden considerarse tres grandes tipos de ciudades que, con poblaciones que oscilan entre los 10.000 habitantes y los 100.000, reúnen algunos de los rasgos característicos de una ciudad media. Son los casos de las ciudades medias que forman parte de las aglomeraciones urbanas, de las que se sitúan en el litoral y de las que se localizan en los ámbitos interiores.

En el primer grupo se encuentran las ciudades incluidas en el área de influencia directa de alguna de las diez ciudades principales andaluzas. Son, por regla general, las de mayor tamaño poblacional. Bastantes de ellas superan los 50.000 habitantes y algunas han alcanzado, incluso, el umbral de los 100.000 . Los ejemplos más significativos están en las aglomeraciones de Sevilla, Bahía de Cádiz-Jerez de la Frontera, Bahía de Algeciras, Málaga y Granada. Normalmente son también las que concentran mayores y más especializados servicios urbanos. Su naturaleza de ciudad media queda, sin embargo, algo desdibujada por su pertenencia a una unidad urbana y territorial superior como son los Centros Regionales: las aglomeraciones urbanas surgidas en torno a las grandes ciudades andaluzas. Estos Centros funcionan ya como una realidad que desborda los límites administrativos municipales y que físicamente puede vi- sualizarse en un continuo urbano. Aunque algunas de estas ciudades medias son auténticas ciudades históricas con una identidad muy marcada, desde un punto de vista funcional muchas han dejado ya de ser ciudades aisladas para pasar a ser una parte más de la aglomeración.

Un caso especial lo representan las ciudades medias del Centro Regional de Bahía de Cádiz-Jerez de la Frontera y del Centro Regional de Bahía de Algeciras: ciudades como San Fernando, Puerto Real, la Línea o el Puerto de Santa María. La naturaleza marcadamente policéntrica que desde siempre han caracterizado a estos Centros hace que estas poblaciones mantengan una identidad propia más acusada y que las funciones que desempeñan mantengan una especialización, que no suele darse en el resto de los Centros, en los que el peso de la ciudad central es muy superior.

Las ciudades medias litorales situadas fuera de los Centros Regionales son, sin duda, de los tres grupos enunciados las que mayor dinamismo relativo han demostrado en las últimas décadas. Muchas de ellas ostentan en estos momentos las tasas de crecimiento demográfico, productivo o de construcción de viviendas más altos de toda la Comunidad Autónoma. No son, salvo algunas excepciones (Sanlúcar de Barrameda, Motril, Almuñécar...) ciudades medias históricas en el sentido de que hasta hace relativamente pocos años no tenían un rango auténticamente urbano. Pero los cambios en las pautas del poblamiento que se han dado en la región y que han favorecido el desplazamiento de la población al litoral y, sobre todo, la expansión de las dos actividades productivas que más han crecido dentro de Andalucía: la agricultura intensiva bajo plástico y el turismo de litoral han provocado un acelerado proceso de urbanización en prácticamente toda la franja costera. Se trata, no obstante, de un tipo de ciudad media especial. El mismo dinamismo a que están sometidas y la peculiar ocupación del espacio que genera el turismo y la agricultura intensiva hacen de estas ciudades un fenómeno singular que rompe con lo que han sido las formas de organización territorial históricamente presentes en Andalucía. Se están creando, en este sentido, verdaderas conurbaciones, como ya sucede en la Costa del Sol, los tejidos urbanos se forman al margen de los pequeños cascos urbanos originarios, los modelos de organización territorial rara vez son concéntricos en estas áreas y en muchas ocasiones es difícil identificar un núcleo como cabecera comarcal.

El tercer y último grupo de ciudades medias son las situadas en el interior rural de la región. Son, en total, unas 60 ciudades de no gran tamaño -casi ninguna alcanza los 50.000 habitantes- que concentran cerca de 1.200.000 habitantes. Suponen sólo el 16\% de la población andaluza, pero su trascendencia territorial es muy superior a la que se pude deducir de esta simple cuantificación demográfica. Una parte muy importante del territorio regional mantiene una dependencia funcional de ellas. Las vegas y campiñas 
interiores del Valle del Guadalquivir y de las depresiones interiores que jalonan el denominado Surco Intrabético tienen en estas ciudades sus únicas referencias urbanas.

\section{LAS “AGROCIUDADES” DEL BAJO GUADALQUIVIR}

Las ubicadas en los tramos inferiores del Valle del Guadalquivir, caso de Écija, han recibido tradicionalmente el nombre de "agrovillas" o "agrociudades". Se trata de unas 15 ciudades con poblaciones que oscilan entre los 10.000 y los 45.000 habitantes que se distribuyen de forma sensiblemente regular por el territorio comprendido entre la ciudad de Córdoba y el litoral. En total concentraban el año 2000 casi

\section{Construcción de viviendas (1994=100)}

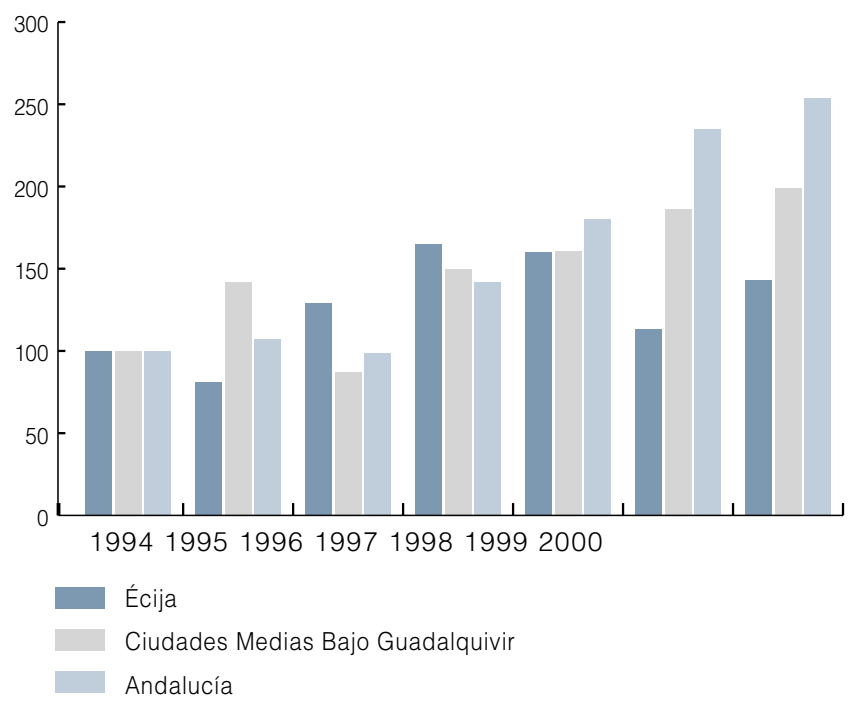

400.000 habitantes, que vienen a representar algo más del 5\% de la población de la región. Curiosamente, salvo alguna excepción, casi todas se localizan en las zonas de campiñas apartadas del cauce del Guadalquivir.

El término agrociudad viene a reconocer la directa relación que han tenido desde siempre estos núcleos de población con la elevada productividad de las fértiles tierras sobre las que se localizan. La riqueza agrícola ha sido la que ha hecho posible el florecimiento de este tipo de fenómenos urbanos, difícilmente concebibles en ámbitos con producciones agrícolas de menor entidad.

Se trata, no obstante, también de una denominación con connotaciones claramente negativas, toda vez que cuestiona el verdadero carácter urbano de estas poblaciones. A pesar del volumen de población residente en ellas muchas veces se ha entendido que más que auténticas ciudades se trataba de grandes concentraciones de población rural que no reunían los requisitos exigibles a una ciudad en cuanto servicios, equipamientos o actividad económica. Hasta cierto punto han sido consideradas, por ello, como una anomalía propia de la región andaluza motivada por unos sistemas de propiedad de la tierra dominados por la gran propiedad latifundista y necesitados de grandes bolsas de jornaleros sin tierras, que sólo desde un punto de vista cuantitativo se podía poner en relación con los auténticos hechos urbanos.

Sea como fuere, y con independencia del hecho de que en estos momentos las ciudades medias andaluzas ya no respondan a este concepto de agrociudad, lo cierto es que, junto al policentrismo que se da en el nivel superior de la jerarquía, esta clase de ciudades es uno de los rasgos que más y mejor singularizan el sistema urbano regional andaluz. El número, las dimensiones y la distribución territorial de ciudades medias que se dan en las vegas, campiñas y depresiones andaluzas son difíciles de encontrar en otras regiones españolas o europeas, donde los ámbitos rurales suelen aparecer ocupados por una población más diseminada o, en todo caso, por núcleos de población de tamaños muy inferiores a los andaluces.

Se trata, además, de una singularidad de carácter histórico, una forma de organización del territorio desde estas ciudades consolidada ya desde hace siglos. El patrón territorial de concentración de la población en un número reducido de asentamientos de mediano tamaño distantes entre sí y la formación en tono a ellos de un número nunca muy elevado de pequeñas poblaciones rurales ha sido una constante en la Historia de Andalucía. Y es por ello que, a diferencia de las ciudades medias de las aglomeraciones urbanas y, de forma más acusada aún, de las litorales, estas ciudades interiores disfrutan hoy de una fuerte identidad histórica. Prácticamente todas han tenido un papel protagonista en la historia urbana, social y política de Andalucía. La mayoría de ellas aparecen ya consolidadas como cabeceras concejiles o señoriales en la Baja Edad Media. Con esta circunstancia hay que poner en relación, entre otras cuestiones, los extensos términos municipales que aún conservan muchas de ellas.

En el caso de Écija la participación en la historia regional se remonta, incluso más atrás, a la Antigüedad. En muchos momentos, además, su protagonismo fue más allá de lo que cabría esperar de una simple cabecera comarcal. Durante la época romana, por ejemplo, la ciudad, emplazada en un punto altamente estratégico de la Vía Augusta como es el paso del río Genil, fue la cabecera de uno de los cuatro conventos jurídicos en que estuvo dividida la provincia de la Bética y, junto a Sevilla y Córdoba, formaba el principal eje urbano del Bajo Guadalquivir, un eje que en gran medida sigue, aún hoy, siendo el principal referente territorial de esta parte de la región. Por su parte, en el periodo islámico fue cabecera de cora y su medina llegó a alcanzar en el siglo XII la considerable extensión de 56 hectáreas 
y una población de aproximadamente 18.000 habitantes que la situaban entre las diez ciudades principales de al-Andalus, por encima de capitales tan significativas como Málaga, Zaragoza o Valencia. Todavía en el siglo XVI, Écija, junto a otras de las actuales ciudades medias andaluzas se encontraba entre las 25 ciudades más pobladas de la Península Ibérica.

La entrada en la Edad Contemporánea supone, no obstante, para la mayoría de las agrociudades el inicio de una prolongada etapa de decadencia. Con más intensidad si cabe que otras partes de Andalucía, estas poblaciones quedaron marginadas de los grandes procesos de industrialización que tienen lugar a lo largo del siglo XIX y gran parte del XX. Volcadas casi exclusivamente en las actividades primarias de producción y ajenas a las nuevas transformaciones sociales y económicas, la evolución de estas agrociudades las aleja progresivamente de las pocas comarcas andaluzas que logran integrarse de forma ventajosa en los nuevos circuitos económicos y de las capitales provinciales, que se ven favorecidas por la ubicación en ellas de los servicios de la Administración que acompañan a la nueva división territorial del Estado.

A este periodo de estancamiento casi secular le sigue un periodo de crisis, que si no puede considerarse especialmente prolongado -las décadas centrales del siglo $X X$, años cincuenta, sesenta $y$ setenta-, sí es, en cambio particularmente intenso. En un corto espacio de tiempo la coincidencia de dos factores: por una parte, la modernización de la agricultura que hace descender radicalmente el trabajo empleado en la producción agrícola; por otra, la fuerte demanda de empleo que se genera en las regiones y ciudades más industrializadas del resto de España y Europa provocan una emigración masiva que desarticula definitivamente la sociedad y las formas de vida que habían caracterizado a estos núcleos urbanos de las campiñas del Guadalquivir.

Con la excepción de los municipios que se vieron favorecidos por la transformación en regadío de la denominada zona regable del Bajo Guadalquivir (casos de Lebrija o de Utrera), casi todas las ciudades medias interiores sufrieron pérdidas muy considerables de población. En el municipio de Écija, por ejemplo, el saldo entre los censos de 1950 y el de 1981 arroja una pérdida absoluta de unos 7.000 habitantes, casi el 5\% de la población censada en 1950. Las implicaciones de todo tipo que tiene una regresión demográfica de esta naturaleza son evidentes; entre otras un envejecimiento relativo de la población municipal y una pérdida de los elementos más cualificados y potencialmente innovadores de la ciudad. Las consecuencias de todo ello fueron la formación de grandes bolsas de paro, una baja diversificación y cualificación productiva y unos déficits relativos en cuanto a equipamientos e infraestructuras que alejaron cada vez más a estas ciudades de las áreas urbanas más desarrolladas de la región y el Estado.

\section{EL PASO DE AGROCIUDAD A CIUDAD MEDIA}

A partir de 1980 y de forma más acusada desde finales de esta década y a lo largo de la siguiente se produce una inflexión de la situación. Hay una recuperación demográfica en todas estas ciudades medias, se producen en ocasiones espectaculares crecimientos del espacio construido y, lo que es más importante mejoran sensiblemente las condiciones de vida y las expectativas de desarrollo económico. En el municipio Écija, que no ha sido uno de los más dinámicos, desde 1981 a 1996 se han recuperado 2.589 habitantes y las tasas de crecimiento poblacional han vuelto a ser netamente positivas, del 0,5\% anual. En viviendas el crecimiento ha sido considerablemente más intenso. El parque se ha incrementado en casi 2.000 viviendas entre 1981 y 1991, un ritmo de construcción ( $1,1 \%$ anual) que se ha acelerado en los últimos años. En concreto, entre 1994 y 2000 se han construido 2.39I viviendas de nueva planta, lo que supone incrementar en tan sólo 6 años el parque municipal en casi un 19\%.

Evolución población 1900 - 1996. (1900=100)

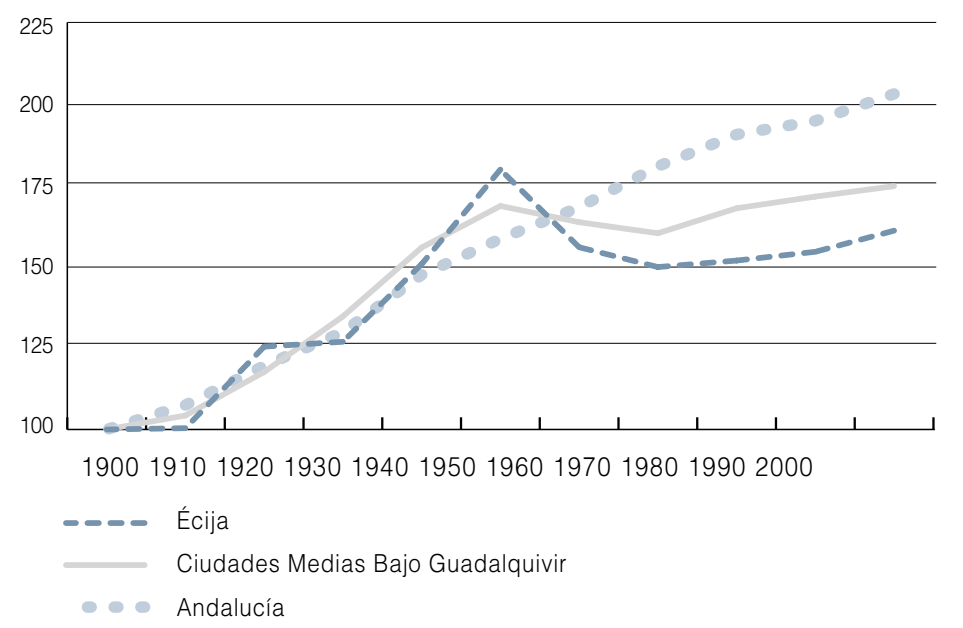

Las causas de esta nueva tendencia son varias y no necesariamente son idénticas para todas las ciudades medias del Bajo Guadalquivir. Hay, no obstante, algunos factores que parecen encontrarse detrás de la recuperación experimentada. En primer lugar los ciclos expansivos generales de la economía andaluza y española en los ochenta y los noventa han alcanzado también lógicamente a estas poblaciones y las economías locales se han visto favorecidas por ello. Pero quizá mayor importancia hayan tenido los efectos provocados por la extensión a las zonas rurales de Andalucía del llamado Estado del Bienestar. La fuerte inversión pública en mejorar la dotación de servicios, equipamientos e infraestructuras en los municipios rurales andaluces ha sido, en este sentido, un factor trascendental para recuperar la centralidad de muchas de las antiguas agrociudades. La implantación en ellos de nuevos servicios y equipamientos de carácter supramunicipal (educativos, sanitarios, culturales, etc.) han reforzado notablemente las funciones de 
cabecera comarcal que históricamente venían desempeñando esta clase de ciudades.

Hay un tercer factor a tener en cuenta igualmente como es la favorable posición de que han disfrutado muchas ciudades medias históricas para aprovechar en beneficio propio el crecimiento experimentado en las grandes aglomeraciones urbanas. Los problemas de saturación que comienzan a darse en estos años en Centros Regionales como el de Sevilla y la insuficiencia en ellos de la oferta de suelo, ya sea éste residencial o productivo, están favoreciendo la reubicación de actividades en las antiguas agrociudades que aún no soportan este tipo de problemas. Por su parte las mejoras introducidas en las infraestructuras regionales, en especial en las grandes redes viarias, han contribuido a disminuir los tiempos de desplazamiento y a hacer posible la formación de estas segundas coronas metropolitanas, que en casos como los de Utrera, Los Palacios o Carmona empieza a ser una realidad.

No debe olvidarse, por último, la aparición de nuevas iniciativas locales, que surgen del propio ámbito rural y que también han desempeñado un papel en la reactivación experimentada. Aún de forma incipiente ya son muchas las actividades industriales o terciarias que surgidas de las propias sociedades locales están contribuyendo a diversificar las economías locales y mejorar su capacidad de competir.

\section{LAS CIUDADES MEDIAS INTERIORES COMO PATRIMONIO TERRITORIAL Y URBANO: LA ORDENACIÓN DEL TERRITORIO Y EL PATRIMONIO HISTÓRICO URBANO}

Con independencia de otras cuestiones el nuevo marco en el que se está desarrollando la vida de estas ciudades está volviendo a poner de manifiesto su carácter de ciudades históricas y, en especial las implicaciones urbanísticas y territoriales que eso conlleva. Quizá la más evidente es la formación de un patrimonio de carácter monumental que se sitúa entre los más ricos de la región y que en estos momentos, posiblemente, haya pasado a ser uno de los mejores recursos para el desarrollo con los que cuentan las poblaciones del interior de Andalucía. Las grandes posibilidades que en este campo aporta este tipo de patrimonio son claras. Es un reclamo de primer orden para un sector emergente como es el turismo cultural, un atractivo para atraer actividades no directamente turísticas, pero que sí reclaman cada vez más unos entornos de calidad (actividades profesionales, servicios especializados, industrias culturales, etc.) y es, en definitiva, un elemento simbólico que contribuye a reforzar las identidades locales y la cohesión social de los municipios.

El aprovechamiento de estas potencialidades no está en cualquier caso exenta de riesgos y requiere una utilización sensible, culta podría el término justo, del recurso patrimonial. Este tipo de patrimonio es cla- ramente un recurso no renovable y como tal insustituible. El concepto de sostenibilidad, originariamente reservado a la explotación de los recursos naturales, ha pasado a ser un lugar común por el abuso en la utilización del término. En este caso, sin embargo, su aplicación práctica más allá de las simples declaraciones de intenciones parece especialmente necesaria para poner en valor este patrimonio con garantía de éxito a largo plazo.

Pero, junto a todo ello, hay otras implicaciones menos llamativas, pero no por eso menos importantes. Una de ellas ya se ha apuntado en parte y está relacionada con el papel que las ciudades medias, en general, y Écija, en particular, tienen en la organización del territorio interior de Andalucía; un papel que puede considerarse como un auténtico patrimonio territorial de la región, en tanto en cuanto es resultado de una determinada forma de estructurar el territorio que históricamente ha demostrado su eficacia. Ciudades como Écija son polos que ordenan amplios territorios rurales alejados de las grandes aglomeraciones metropolitanas, que gracias a ello pueden mantener unos mínimos niveles de integración en el sistema urbano regional. La tradición que acumulan como cabeceras comarcales de ámbitos rurales son, en este sentido, un valor que se debe preservar y aprovechar para mejorar la competitividad regional andaluza.

El valor territorial de estas ciudades históricas ha sido plenamente reconocido por la ordenación del territorio regional. Una de las principales líneas argumentales del documento de Bases y Estrategias del Plan de Ordenación del Territorio de Andalucía es precisamente potenciar las ciudades medias interiores como elementos útiles para estructurar un territorio, tan problemático, por otra parte, en este aspecto como es el andaluz.

Esta potenciación conlleva, no obstante, algo más que una simple política de implantación de equipamientos e infraestructuras supramunicipales. En buena medida esto último se ha realizado ya y los avances son evidentes. Es fundamental, además, que se supere la percepción tradicional de la ciudad media interior como agrociudades aisladas en su ámbito territorial inmediato. En otras palabras, es imprescindible que se formen redes supramunicipales de ciudades medias que multipliquen la competitividad de núcleos de población que aisladamente tienen limitadas sus posibilidades de progresar. Esto implica forzosamente mejorar la cooperación entre los municipios y entre los respectivos agentes sociales, institucionales y económicos; y es cierto que, en este aspecto concreto, la herencia histórica no ha contribuido a favorecer relaciones de complementariedad, más bien al contrario, lo más habitual hasta fechas relativamente recientes ha sido la competencia. Si hablar en estos momentos de redes de ciudades en las aglomeraciones urbanas o en las franjas más dinámicas del litoral andaluz es referirse a una realidad incipiente, hacerlo de las ciudades medias del interior tiene, todavía, mucho de voluntarismo territorial. 
Hay, a pesar de ello, algunos hechos que permiten ser optimistas en esta cuestión. De hecho, en los últimos años las iniciativas de cooperación han crecido de forma espectacular. Las mancomunidades y consorcios municipales se han multiplicado, la formación de los denominados Grupos de Acción Local (grupos PRODER y LEADER) se han extendido por todas las zonas rurales e, incluso, ya se han puesto en marcha valiosas iniciativas de puesta en valor conjunto del patrimonio cultural. Es cierto que algunas de ellas se encuentra el efecto indirecto de las exigencias de la Unión Europea para distribuir sus ayudas al desarrollo regional y, en ocasiones, la cooperación es sólo formal y administrativa, pero, en cualquier caso implica un avance sustancial que cabe esperar que se consolide.

En otro sentido el carácter histórico de las ciudades medias interiores ha dejado unos centros históricos que no deben identificarse exclusivamente con la mera suma de edificios monumentales, ni tampoco como simples lugares singulares. Se trata de tejidos urbanos de un valor histórico trascendental, muestra de una forma de ocupación del espacio que debe ser objeto específico de protección con independencia de que hayan sido favorecidos, o no, con una declaración administrativa de conjunto histórico.

El peso de estos centros históricos dentro de las ciudades actuales es aún muy alto. A diferencia de lo que ocurre en las grandes capitales en las que los crecimientos urbanos han adquirido unas dimensiones tales que lo hacen en cierta forma independientes de los cascos históricos originarios, en las ciudades medias la mayor parte del suelo urbano sigue siendo ciudad histórica y los desarrollos recientes, al margen de su mayor o menor acierto, han estado muy condicionados por la ciudad preexistente. Los crecimientos futuros tendrán que tener en cuenta necesariamente esta circunstancia, ya que su incidencia en la ciudad histórica será determinante en muchos aspectos.

En este sentido, dos parecen los retos que tienen que enfrentar los centros históricos de estas ciudades medias. El primero es superar una concepción de lo histórico limitado a lo monumental y, en su caso, a lo pintoresco. La protección que requieren, ya venga ésta derivada de la legislación patrimonial o del pla- neamiento urbanístico o territorial, debe ir más allá de la preservación de las construcciones singulares o del mantenimiento estético de fachadas, aspectos algunas veces no sustanciales de la cuestión. La protección debe ser, en definitiva, estructural e integral y debe tener como objetivo la conservación de los valores de cada casco y su recuperación como piezas vivas dentro de la ciudad. Una recuperación que, por otra parte no debe interpretarse en términos sólo económicos. Evitar una excesiva terciarización de los centros de las ciudades y mantener un volumen alto de población residente debe ser uno de los objetivos a alcanzar.

La reactivación de estos espacios no debe, sin embargo, llevar a reproducir modelos implantados en las grandes capitales que ya han vivido fuertes procesos de crecimiento y que hoy están demostrando sus carencias. Las ciudades medias tienen, en relación con esto, una importante oportunidad para buscar otro tipo de soluciones más imaginativas a sus nuevos problemas y evitar caer en errores ya cometidos en otros ámbitos.

Puede que el tratamiento del tráfico sea uno de los ejemplos más claros de todo esto. Evidentemente se trata de una cuestión compleja que no puede resolverse como medidas simples. La acelerada motorización que esta viviendo nuestra sociedad es un hecho de tal envergadura que no debe, en ningún caso, minusvalorarse. Pero sí parece claro que deben imponerse algunos objetivos y prioridades. Una de ellas es la que se deriva de la constatación de que las tensiones que produce el tráfico privado hacen imposible una mínima calidad de vida en centros históricos que físicamente no pueden acoger un volumen de tráfico que crece de forma exponencial. Calmar el tráfico, favorecer el transporte público, liberar espacios para los peatones son medidas cada vez más imprescindibles que chocan con intervenciones que incentiven el traslado al centro en vehículos privados. La política de aparcamientos es en este aspecto fundamental. Soluciones de difícil o costosa ejecución en las grandes ciudades son todavía viables en las ciudades medias y todas parecen pasar por evitar incrementar las plazas de aparcamientos rotatorios en los puntos centrales de la ciudad y favorecer alternativas de lo que podría llamarse una movilidad sostenible y responsable. 